\title{
GRADUAÇÃO TECNOLÓGICA NO BRASIL E NA FRANÇA: EM BUSCA DE UM PERFIL IDENTITÁRIO DENTRO DA RELAÇÃO ENSINO/TRABALHO NAS INSTITUIÇÕES DE ENSINO SUPERIOR BRASILEIRAS
}

\author{
Marise Miglioli Lorusso \\ UNIMEP - Universidade Metodista de Piracicaba \\ marise.unimep@gmail.com 1 \\ Dalila Alves Correa \\ UNIMEP - Universidade Metodista de Piracicaba \\ dacorrea@unimep.br
}

\section{RESUMO}

Este trabalho apresenta os dados preliminares da pesquisa sobre o perfil identitário das graduações tecnológicas no Brasil (SENAI, FATEC e outros) e na França (CNAM), com ênfase à metodologia de implantação de cursos, o mercado de trabalho e com o perfil dos ingressantes/egressos. A proposta final é a de apresentar um modelo analítico a partir do estudo comparativo do tratamento dos cursos superiores de tecnologia nas instituições brasileiras e na França. A abordagem da pesquisa é qualitativa e utiliza a metodologia de casos múltiplos, utilizando técnicas de entrevistas semiestruturadas, observação e análise documental para triangulação de dados. As entrevistas serviram para apontar novas dimensões do objeto de estudo e tem se constituído como fator determinante dos rumos do trabalho. Os resultados obtidos até o momento ainda são inconclusivos e apontam para uma indefinição da identidade dos cursos de graduação tecnológica em relação ao seu propósito de implantação. A legitimação do status do curso carece de sustentação argumentativa para seu objetivo primordial. A conclusão prévia a partir dos dados parciais sugere a necessidade de um modelo analítico que alicerce a definição do perfil identitário dos cursos no Brasil a partir do modelo francês.

PALAVRAS-CHAVES: Administração - Ensino e Pesquisa; SENAI - Educação Superior; FATEC Educação Superior; CNAM (Consérvatoire National des Arts et Métiers) - Educação Superior; Graduações Tecnológicas (Brasil) 


\section{Abstract}

This paper presents the preliminary data of the research on the identitary profile of technological graduations in Brazil (SENAI, FATEC and others) and in France (CNAM), with emphasis on the methodology of course's implementation, the labor market and the profile of the students / Graduates. The final proposal is to present an analytical model based on the comparative study of the treatment of higher technology courses in Brazilian institutions and in France. The research approach is qualitative and uses the methodology of multiple cases, using techniques of semi-structured interviews, observation and documentary analysis for data triangulation. The interviews served to point out new dimensions of the object of study and has been constituted as a determining factor of the directions of the work. The results obtained so far are still inconclusive and point to a lack of definition of the identity of technological graduation courses in relation to their purpose of implementation. The legitimation of course status lacks argumentative support for its primary purpose. The previous conclusion from the partial data suggests the need of an analytical model that bases the definition of the identity profile of the courses in Brazil from the French model.

KEYWORDS: Administration - Teaching and Research; SENAI - Higher Education; FATEC - Higher Education; CNAM (Consérvatoire National des Arts et Métiers) - Higher Education; Technological Graduations (Brazil)

\section{INTRODUÇÃO}

Os cursos superiores de tecnologia existem, no Brasil, desde a década de 60 , tendo por objetivo precípuo a formação do profissional voltada para o mercado de trabalho, com competências para lidar com as situações cotidianas das organizações (GONÇALVES, 2010). Essa relação educação/mercado de trabalho vem sendo discutida em eventos voltados ao mercado de trabalho e à produção de conhecimentos significativos que possam gerar inovações, principalmente na área tecnológica, dentro das organizações como um todo.

Contudo, a grande massa de egressos das diversas instituições de ensino superior já não está mais voltada à questão técnica e tecnológica. Pelos dados do MEC/SESU, percebe-se o crescimento dos cursos voltados às áreas humanistas, transformando as graduações regulares em cursos de menor duração.

Neste pormenor, há diversos questionamentos que se impõem: o fato da tecnologia enquanto ferramenta ter uma participação fundamental na vida das pessoas não estaria interferindo na duração de um curso regular? Por outro lado, a crescente rede informacional não exigiria cargas horárias condizentes com a necessidade de compartilhar um número razoável de conhecimentos para a geração de possíveis inovações?

E no final do caminho, surge uma pergunta considerada fundamental para a pesquisa: qual é o real perfil desses cursos, sua identidade e sua relação identitária com o mercado de trabalho?

Com base nesse questionamento, o trabalho final pretende propor um modelo analítico que identifique do perfil das graduações tecnológicas no Brasil, com base no modelo educacional do CNAM (França).

A relevância do tema está centrada nas possíveis contribuições à Educação Superior, onde tantas instituições oferecem cursos de graduação tecnológica se necessariamente avaliar sua viabilidade ou identificar o perfil do curso a ser ofertado. A escolha, diversamente da 
adequação de um perfil a uma vaga de emprego, aparenta pautar-se na oportunidade de oferta e na demanda pela população.

Trata-se de utilizar as vias de resolução apontadas por Sainsaulieu (1997), onde uma norteia para a evolução através do trabalho e a outra, pela simbiose entre indivíduo empresa.

Na primeira, a identidade revela-se pelo fato do curso conduzir à evolução enquanto a segunda direciona para as expectativas de crescimento por meio de um aperfeiçoamento básico de curta duração que remeta o indivíduo aos objetivos imediatos para sua carreira.

O estudo de viabilidade apresenta incongruências cujas pesquisas deverão apontar, bem como a proposta de um modelo para a viabilidade face às discrepâncias notadas entre o modelo atual e o modelo proposto.

\section{DESENVOLVIMENTO}

Durante a pesquisa de literatura sobre graduações tecnológicas de curta duração, principalmente em seus aspectos legislativos, pôde ser observado que a ênfase parece voltada aos aspectos de ingresso e duração dos cursos, pouco se comentando sobre a metodologia de implantação ou com a visão de longo prazo.

Não se encontram, de forma pormenorizada, dados referentes aos resultados acerca de possíveis impactos no mercado de trabalho. À primeira vista, parece que o Brasil tem necessidade absoluta de quantificar números de pessoas com formação superior para alterar sua posição do ranking mundial. Em análise mais profunda, podem ser verificados aspectos mais ligados às questões sociais. Contudo, estudos em profundidade que não se limitem às pífias explanações sobre o preparo para o mercado de trabalho, bem como estudos que se proponham a analisar conhecimentos e a necessária geração de inovações dentro das organizações não são elaborados com muita frequência.

Assim, já que o modelo francês tem se apresentado como uma questão viável e uma vez que o modelo analítico a ser proposto estabelece uma comparação entre o modelo brasileiro e o modelo francês, julgou-se oportuno optar pelo viés dos resultados desses perfis dentro das organizações, sob a ótica do conhecimento que, por sua vez, gera poder dentro das próprias organizações.

\subsection{GRADUAÇÕES TECNOLÓGICAS NO BRASIL}

No panorama apresentado pelo MEC (Ministério da Educação), em seu Catálogo Nacional dos Cursos Superiores de Tecnologia (2010), existem 112 graduações tecnológicas organizadas em 13 eixos tecnológicos, com as respectivas denominações, sumário de perfil do egresso, carga horária mínima e infraestrutura.

Entre tanta diversidade, cabe aqui a ênfase aos cursos voltados ao Eixo dos Pricessos Gerenciais, apresentados no mesmo Catálogo, que incluem: Empreendedorismo e Gestão de Negócios; Empreendedorismo; Empreendimento; Gerência de Processos Empresariais; Gestão Bancária; Gestão da Informação em Negócios; Gestão de Agências Bancárias; Gestão de Bancos e Finanças; Gestão de Bancos e Mercado Financeiro; Gestão de Empreendimentos Educacionais; Gestão de Empreendimentos Rurais; Gestão de Empreendimentos; Gestão de Empresas de Serviços; Gestão de Instituições Financeiras; Gestão de Médias e Pequenas Empresas; Gestão de Micro e Pequenas Empresas; Gestão de Negócios de Pequeno e Médio Porte; Gestão de Negócios e da Informação; Gestão de Negócios e Empreendedorismo; Gestão de Negócios em Comércio e Serviços; Gestão de Negócios em serviços Terceirizáveis; Gestão de Negócios Empresariais; Gestão de Negócios; Gestão de Pequenas e Médias 
Empresas; Gestão de Pequeno e Médio Empreendimento; Gestão de Processos Empreendedores; Gestão de Processos Organizacionais; Gestão e Desenvolvimento de Sistemas Corporativos; Gestão Empreendedora da Informação; Gestão Empreendedora de Empresas; Gestão Empreendedora de negócios; Gestão Empreendedora de Pequenos Negócios; Gestão Empreendedora; Gestão de Negócios; Gestão Empresarial de Pequenas e Médias Empresas; Gestão Empresarial e Tecnologia da Informação; Gestão Empresarial; Gestão Estratégica de Organizações; Gestão Estratégica de Pequenas e Médias Empresas; Gestão Estratégica Organizacional; Gestão Executiva de Negócios; Pequenas Empresas; Planejamento Administrativo e Programação Econômica.

Lembrando que a gama de cursos acima remete às disciplinas contidas nos Cursos de Administração, questiona-se a metodologia que as Instituições de Ensino utilizaram para compor seus currículos, definir os reais objetivos de tais cursos e o acompanhamento de seus egressos.

Até o ano 2000, conforme demonstrado pela legislação nacional, havia significativa diferença entre a proposta das graduações tecnológicas e a dos cursos de bacharelado e licenciatura vigentes no Brasil.

Os primeiros, eram geralmente destinados à ocupação imediata dos postos de trabalho de supervisão e/ou gestão das indústrias, aliando a capacidade técnica de execução de tarefas ao gerenciamento de processos, por "tecnólogos" ou "técnicos de nível superior".

Com a evolução de sistemas automatizados, derivados da ampliação do uso da INTERNET ou da importação de tecnologias necessárias à competitividade das empresas em cenário de globalização, o aperfeiçoamento dos processos e o perfil do profissional para gerenciá-los tornaram-se alvo de estudos e debates por parte das empresas e das instituições de ensino.

Contudo, a amplitude desse debate e os dados obtidos a partir do mesmo só estão evidenciados nos relatórios oficias do SENAI e da FATEC. ${ }^{1}$

Por ser mantido e administrado pela indústria, o SENAI estabelece, de forma sistemática, um intercâmbio de informações entre as partes envolvidas, a fim de poder traçar as diretrizes de seus cursos superiores, a exemplo dos procedimento consolidados para os níveis de aprendizagem e técnicos.

\subsection{CURSOS SUPERIORES DE TECNOLOGIA SENAI}

Os cursos superiores de tecnologia oferecidos pelas Faculdades SENAI-SP correspondem à educação profissional de nível tecnológico, descrita na legislação educacional brasileira vigente, conforme o Relatório de Atividades do SENAI (2012). O ingresso é feito pela modalidade de exame de seleção após obrigatória conclusão do Ensino Médio.

De acordo com o mesmo Relatório, existem 13 (treze) graduações tecnológicas, que obtiveram um crescimento de $20,2 \%$ de matrículas, quando estabelecida uma comparação entre 2011 e 2012.

Em entrevista realizada com o Diretor da primeira Escola SENAI a contemplar um Curso Superior, foi enfatizada a "estreita relação entre a implantação do Curso e a necessidade do mercado de trabalho". A sincronizada relação entre empregado/empregador/mantenedor, oferece uma visão realista dos componentes econômicos, sociais e políticos que envolvem a curto, médio e longo prazo, os destinos de determinadas profissões. Tal metodologia permite a criação segura de novos cursos, com absorção mercadológica viável, atualização permanente e desenvolvimento de competências, de acordo com as necessidades.

\footnotetext{
${ }^{11}$ N.A. Neste ponto da pesquisa ainda não foi concluído o estudo do CNAM pela autora.
} 
O perfil do docente, do aluno e do mercado que dependerá do resultado que ambos obtiverem no desenvolvimento de habilidades e competências.

A transferência de tecnologia é fato consumado, tornando-se a escola um ambiente que procura reproduzir, da forma mais próxima possível, o ambiente, a tecnologia e o comportamento esperado dos egressos nos postos de trabalho que ocuparão na indústria.

Isso implica em acurado estudo para a abertura e manutenção de novos cursos, para que os mesmos se justifiquem em termos de investimento e resultados.

\subsection{CURSOS SUPERIORES DE TECNOLOGIA FATEC}

Anteriormente à criação de Cursos Superiores pela iniciativa privada, o Governo do Estado de São Paulo criou as Faculdades de Tecnologias (FATECs), cujos egressos buscavam uma profissão ao invés de uma continuidade acadêmica de pesquisa teórica ou assemelhada.

As FATECs são mantidas pelo Centro Paula Souza, uma autarquia do Governo do Estado de São Paulo vinculada à Secretaria de Desenvolvimento Econômico, Ciência, Tecnologia e Inovação, com 59 unidades em 162 municípios paulistas, com mais de 64 mil alunos matriculados nos 65 cursos de graduação tecnológica, conforme informações do site oficial da Instituição.

O marco inicial da trajetória das FATECs foi a fundação, em 1969, do Centro Estadual de Educação Tecnológica de São Paulo que tinha por objetivo a formação de técnicos de nível superior para atender a crescente demanda de profissionais de nível universitário. Os cursos ministrados pela FATEC-SP são os mais antigos, tendo sido ministrados ininterruptamente desde 1970.

Em 1973, por meio de decreto estadual, o Centro passou a ser denominado Centro Estadual de Educação Tecnológica Paula Souza e seus cursos passaram a constituir a Faculdade de Tecnologia de São Paulo. Dessa forma, o CEETEPS se tornou o mantedor de duas FATECs, uma na cidade de São Paulo e a outra em Sorocaba. Em 1974, foi criado o Curso Superior de Tecnologia em Processamento de Dados, até hoje referência dentre os cursos de tecnologia na área de Informática.

À semelhança do que é feito pelo SENAI, o ingresso de candidatos é feito através de processo seletivo, conforme a legislação educacional brasileira.

\subsection{CONTRASTES}

No Brasil, o SENAI e a FATEC destacam-se em um cenário repentinamente voltado para a criação de cursos de graduação sequenciais/tecnológicos, que migraram dos cursos de graduação regulares, tanto devido às dificuldades econômicas de manutenção de custos e salários por parte das instituições de ensino particulares, como pela necessidade de acelerar o engajamento de egressos do ensino médio, aumentando-lhes o potencial de empregabilidade.

Contudo, cabe a reflexão acerca do fato das instituições que constituem objeto desta pesquisa atuarem em consonância e diálogo constante entre as duas pontas do processo educacional. Isso significa dizer que as instituições de ensino superior, diversamente da metodologia utilizada pelo SENAI e pela FATEC, apresentam poucas evidências de diálogo com os polos de empregabilidade ao criarem seus cursos. Uma quantidade considerável de egressos portadores de diplomas de qualificação tem sido alvo de análise e avaliação pelos órgãos normativos da Educação Brasileira, muito embora, a abertura, constituição e autorização para funcionamento de um estabelecimento de ensino possa ser facilmente estabelecida desde que os requisitos mínimos sejam atendidos.

À semelhança dos dados amplamente noticiados pela imprensa local brasileira de que "a cada dez empresas abertas, oito fecham as portas em menos de um ano" (SEBRAE, 2011), 
por falta de estrutura, planejamento estratégico e conhecimento do próprio negócio, as instituições de ensino superior passam a funcionar por estarem de acordo com as normas mínimas estabelecidas. Em visão estratégica míope do próprio negócio, muitas vezes gerado e gerenciado por investidores voltados à rapidez de retorno financeiro, os cursos acabam abrindo e fechando como as empresas.

O reflexo dessa visão estreita pode ser observado no nível de egressos que, efetivamente, encontram trabalho na área de formação, com aplicação dos conteúdos aprendidos.

\section{METODOLOGIA}

Para fins desta pesquisa, de abordagem qualitativa, optou-se pelo estudo de casos múltiplos, a fim de que a comparação entre os mesmos venha a subsidiar a proposta do modelo analítico, triangulados com entrevistas e análise do conteúdo de textos e informações oficiais ou noticiosas.

A partir do estudo em profundidade dos aspectos normativos e legislativos que norteiam as graduações nos modelos brasileiro e francês, são utilizadas as seguintes técnicas investigativas:

a. entrevista semiestruturada com os responsáveis pelas instituições ou aqueles por estes designados, incluindo diretores, ex-diretores, funcionários, instrutores, professores alunos e ex-alunos;

b. questionário para amostra de $10 \%$ dois egressos dos últimos 5 anos, respeitando-se a proporção de formando de cada escola;

c. levantamento, a partir dos dados oficiais de cada instituição, dos segmentos empresariais que absorveram os egressos;

d. análise comparativa da metodologia de cada unidade-caso para implantação de cursos;

g. apresentação do modelo analítico.

\section{CONSIDERAÇÕES FINAIS}

Diversas contradições têm sido verificadas entre a proposta original das graduações e seu estágio atual de evolução. Se havia uma tentativa de encaminhar os egressos ao mercado profissional e/ou ao mestrado profissional, hoje se encontram extremamente emaranhados os tênues fios entre mestrados acadêmicos, mestrados profissionais, sem uma diretriz capaz de estabelecer os limites entre um caminho e outro.

Quando comparado ao modelo francês, ficam evidenciadas questões referentes à linha mestra da Educação no país.

Ainda que a União Europeia possua diferenciais, há alguns casos brasileiros que tentaram aperfeiçoar-se em função dos modelos europeus. O SENAI (Serviço Nacional de Aprendizagem Industrial) é um exemplo, conforme seu histórico e documentação o demonstram. As graduações tecnológicas do SENAI, bem como seu Ensino Técnico e de Aprendizagem (que não são objeto desta pesquisa) orientam-se pela aplicabilidade no mercado profissional, de acordo com uma sequência de ensino que se lança pouco a pouco, após exaustivas pesquisas ligadas à indústria brasileira.

Seguindo o exemplo do SENAI, o Governo do Estado de São Paulo constituiu suas escolas técnicas, voltadas a atingir um público que, na ocasião, não desejava seguir a carreira 
acadêmica. À época de sua implantação, os segmentos acadêmicos e profissionais eram praticamente dois rumos paralelos, sem convergência, a não ser na conclusão de um ensino médio destinado ao atendimento de postos de trabalho.

Nos anos de 1980, conforme boletins de notícias do próprio SENAI, havia uma tendência em "unificar" as escolas do SENAI e as Escolas Técnicas do Governo. Após vários movimentos contrários, dos quais participaram alunos, simpatizantes, dirigentes e uma grande camada da população, optou-se pela não "estatização" do SENAI.

Esta pesquisa, ainda em sua fase preliminar, procurou apresentar dados parciais sobre o estudo das graduações tecnológicas no Brasil e na França, com ênfase aos Cursos de Gestão e Administração, utilizando os modelos metodológicos de concepção, implantação e acompanhamento das graduações tecnológicas do SENAI - Serviço Nacional de Aprendizagem Industrial, FATECs (Faculdade de Tecnologia do Centro Paula Souza - São Paulo) e CNAM (França) nos últimos cinco anos.

As reflexões ainda estão na fase brasileira da pesquisa e parecem apontar a questão metodológica na concepção dos cursos como o diferencial das instituições estudadas.

O estudo do CNAM (França) oferecerá um modelo comparativo para análise de legislações e estratégias em culturas diferentes, bem como a relação da empregabilidade com a constituição dos cursos tecnológicos na França.

Espera-se que os casos estudados possam constituir em reflexão sobre a situação atual do ensino e da pesquisa nos cursos de Administração, tendo em vista a multiplicação acentuada, no Brasil, das graduações tecnológicas. Também aqui o termo "tecnológico", usado para determinar "técnicas", "operações" com uso de tecnologias automatizadas ou não, cede espaço para "cursos de curta duração" na maioria das instituições de ensino brasileiras.

\section{REFERÊNCIAS}

ALBUQUERQUE, Eduardo da Mota e. Questões sobre o trabalho e o valor na era da informática. Belo Horizonte: Mimeo, abril de 1992, 37 p.

BARATO, Jarbas Jovelino. Educação profissional: saberes do ócio ou saberes do trabalho? São Paulo: Editora SENAC, 2004.

BASTOS, J.A.S.L.A. Cursos superiores de tecnologia: avaliação e perspectivas de um modelo de educação técnico-profissional. Brasília: SENETE-MEC, 1991.

BAZZO, Walter Antonio. Ciência, tecnologia e sociedade e o contexto da educação tecnológica. Florianópolis: Edufsc, 1998.

BENCHEIKH, Touhami. Construit social et innovation technologique. Sociologie du Travail, Paris, n. 1, p. 41-57, 1986.

BERGER FILHO, Ruy Leite. Educação profissional no Brasil: novos rumos. Revista Iberoamericana de Educación, n. 20, maio/ago. 1999.

BRASIL. Ministério da Educação e Cultura. Departamento de Assuntos Universitários. Cursos superiores de tecnologia. Brasília: MEC-DAU, 1974.

Decreto $\mathbf{n}^{\mathbf{0}}$ 5.773, de 9 de maio de 2006. Dispõe sobre o exercício das funções de regulação, supervisão e avaliação de instituições de educação superior e cursos 
superiores de graduação e sequenciais no sistema federal de ensino. Disponível em: http://portal.mec.gov.br/setec/arquivos/pdf/dec_5773_06.pdf

Lei $\mathbf{n}^{\mathbf{0}} \mathbf{5 5 4 0 / 6 8}$, de 28 de novembro de 1968. Fixa normas de organização e funcionamento do ensino superior no Brasil. Diário Oficial da República Federativa do Brasil, Rio de Janeiro, 1968.

BRASIL. Ministério da Educação. Secretaria de Educação Profissional e Tecnológica. Catálogo Nacional dos Cursos Superiores de Tecnologia, 2010. Disponível em: www.mec.gov.br.

Brasília: Secretaria de Educação . Educação profissional: legislação básica. 6. ed. Profissional e Tecnológica, 2005. 368 p.

CONSELHO FEDERAL DE ENGENHARIA, ARQUITETURA E AGRONOMIA. Confea. Resolução n⿳ 313/86. Dispõe sobre o exercício profissional dos Tecnólogos das 29 áreas submetidas à regulamentação e fiscalização instituídas pela Lei nº 5.194, de 24 DEZ 1966, e dá outras providências. Disponível em: http://200.242.128.189/legislacao/r313.htm

Resolução no 1.010/ 2005. Dispõe sobre a regulamentação da atribuição de títulos profissionais, atividades, competências e caracterização do âmbito de atuação dos profissionais inseridos no Sistema Confea/Crea, para efeito de fiscalização do exercício profissional. Disponível em: http://www.creapr.org.br/crea/pub/visualizaArquivo

COUNCIL OF EUROPE. The Europe of cultural cooperation: Bologna Process. 2004. Disponível em: http://www.coe.int/T/E/Cultural_Cooperation/education/Higher_education/Activities/Bologna _Process/default.asp.

DECLARAÇÃO da Conferência Mundial sobre Ciência e o Uso do Conhecimento Científico. Santo Domingo, 10-12 mar. 1999. Disponível em: http://www.unesco.org.br/publicacoes/copy_of_pdf/decsantodomingo.pdf

DECOMPS, Bernard; MALGLAIVE, Gérard. Comment asseoir le concept d'université professionnelle? In: BARBIER, Jean-Marie. Savoirs théoriques et savoirs d'action. Paris: Presses Universitaires de France, 2011.

FIDALGO, F.A. A formação profissional negociada: França e Brasil anos 90. São Paulo: Anita Garibaldi, 1999.

GIOLO, Jaime. A educação tecnológica superior no Brasil: os números de sua expansão. Belo Horizonte: Mimeo. 2006.

GONÇALVES, Rafael; PETEROSSI, Helena Gemignani. Os tecnólogos e o mestrado profissional do CEETEPS.

LARUELLE, François. Para o conceito de "não-tecnologia". In: ARAÚJO, H. R. Tecnociência e cultura: ensaios sobre o tempo presente. São Paulo: Estação Liberdade, 
1998. pp. 209-225.

LATOUR, Bruno. Science in action: how to follow scientists and engineers through society. Cambridge, MA: Harvard University Press. 1987.

LIMA FILHO, Domingos Leite. Formação de tecnólogos: lições da experiência, tendências atuais e perspectivas: estudo de caso do Cefet-PR. Boletim Técnico do Senac, Rio de Janeiro, v. 25, n. 3, set./dez. 1999.

MACHADO, Lucília. O profissional tecnólogo e sua formação. Revista da RET - Rede de Estudos do Trabalho, v. 2 p. 20, 2008.

PERRIN, Jacques. La technologie comme science de la technique. In: Comment naissent les techniques: la production sociale des techniques. Paris: Editions Publisud, 1988. pp. 37-55.

PETEROSSI, Helena Gemignani. O tecnólogo e o mercado de trabalho: acompanhamento dos alunos egressos da FATEC -SP. São Paulo: FAT, 1999.

Educação e mercado de trabalho: análise crítica dos cursos de tecnologia. São Paulo: Loyola, 1980.

PRADO, Fernando Leme. Projeto transdisciplinar para graduações tecnológicas com apoio de EAD. Congreso Internacional de Inovación Docente. Barcelona, 28-30 marzo de 2007.

RIBEIRO, Renato J. O mestrado profissional na política atual da CAPES. Revista Brasileira de Pós-Graduação, v. 2, n. 4. p.8-16, jul. 2005.

Ainda sobre o mestrado profissional. Revista Brasileira de Pós-Graduação, v. 3, n. 6. p. 313-315, dez. 2006.

ROSETTI JÚNIOR, Hélio. Tecnólogos e a legislação. Disponível em http:// WWW.idance.com.br/ulbra/legislação_tecnologia.htm. Acesso em 13 de outubro de 2012.

SAINSAULIEU, Renaud. L'identité au travail : les effets culturels de l'organisation. Paris : Presses de la FNSP, 1977.

SAINSAULIEU, Renaud . Apprentissage culturel dans le travail. Psychologie du travail, Paris : Éditions EME, 1978.

SANT'ANDRÉ, Roberto M. Algumas considerações sobre cursos superiores de curta duração - seqüencial e tecnológica. Rio de Janeiro: Paidéia, s.d.

SEBRAE. Empreendedorismo. 2011 (Material didático)

SCHWARTZMAN, Simon. A sociedade do conhecimento e a educação tecnológica. Trabalho realizado por solicitação do Serviço Nacional de Aprendizagem Industrial - SENAI, Departamento Nacional. Janeiro de 2005. Disponível em: www.schwartzman.org.br/simon/pdf/2005_senai.pdf 
TAKAHASHI, Adriana Roseli Wünsch. Cursos superiores de tecnologia em gestão: reflexões e implicações da expansão de uma (nova) modalidade de ensino superior em administração no Brasil. RAP - Revista de Administração Pública. Rio de Janeiro 44(2):385-414, mar./abr. 2010.

UNESCO. A ciência para o século XXI: uma visão e uma base de ação. Declaração da Conferência Mundial sobre Ciência e o Uso do Conhecimento Científico. Santo Domingo, 10-12 mar. 1999. Disponível em:

http://www.unesco.org.br/publicacoes/copy_of_pdf/decsantodomingo.pdf

ZAKON, Abraham. Cientistas, engenheiros, técnicos e tecnólogos: uma questão nova e discutível. Opinião. Adufrj- Seção Sindical, Rio de Janeiro, p. 6-7, 19 de maio de 2003. Disponível em: http://www.eq.ufrj.br/docentes/zakon/questaodiscutivel.pdf 